\title{
Process generalization and the prediction of performance on mental imagery tasks
}

\author{
BENJAMIN WALLACE and BARBARA GERBOC HOFELICH \\ Cleveland State University, Cleveland, Ohio
}

\begin{abstract}
Kosslyn $(1980,1983)$ theorized that performance measures on imagery tasks may vary as a function of the existence of independent processes in imaging ability. The present study determined whether improvement can be made in performance on such tasks with practice. It also considered whether performance on such tasks improves with practice and whether this improvement generalizes. Experiment 1 determined whether improvement in a mental rotation task generalizes to improvement in a geometric analogies task, with both tasks weighted in Kosslyn's find process, but not in a line drawing memory task weighted in Kosslyn's regenerate process. In Experiment 2, we examined generalization in improvement from a geometric analogies task to a mental rotation task. In Experiment 3, we tested whether improvement in an animal imagery task (Kosslyn, 1975) generalizes to improvement in a line drawing memory task, with both tasks weighted in Kosslyn's regenerate process, but not to improvement in a mental rotation task. Performance improved with practice on all tasks. Furthermore, performance improved from one task to another only if both tasks loaded on the same process.
\end{abstract}

Kosslyn (1983) and Kosslyn, Brunn, Cave, and Wallach (1984) have proposed that imagery is a collection of separate abilities involving structures and processes. Structures are used to represent the imagery information and are the medium on which the representations are conveyed. They also include stores of information about the representation, and they make up the visual buffer of Kosslyn's (1983) model. In addition, structures include the spatial, resolution, and granular properties of the medium. Spatial properties involve the size and centeredness of the objects in the visual buffer and determine how much of the image can be seen at one time. Resolution properties determine the clarity and detail of the imagined object, and granular properties determine the minimal size at which the image can be seen so that the fine detail can still be distinguished.

Processes, on the other hand, form patterns of the image on the visual buffer. These patterns are representations formed from information stored in long-term memory (LTM). This stored information includes images, literal memories, and descriptions. Kosslyn, Reiser, Farah, and Fliegel (1983) also proposed that this information is stored in fragments or bits of the whole picture, which allows for the possibility of conjuring up a literal image in its entirety. It can also produce an image that is not exactly literal, such as the image of an elephant on a skateboard.

\footnotetext{
Victor Higgins, Mary Swift, and Jeannie Wareham are gratefully acknowledged for their assistance with data collection and analyses. We also wish to thank Margaret Jean Intons-Peterson and three anonymous reviewers for their many helpful suggestions on an earlier draft of the manuscript. Requests for reprints should be addressed to B. Wallace, Department of Psychology, Cleveland State University, Cleveland, $\mathrm{OH} 44115$.
}

Kosslyn et al. (1984) refer to these processes as specific modules in the processing system, each comprising its own function and operation. Together, these modules form the complex processing system in imagery. Two of the operations in Kosslyn's $(1980,1983)$ model are called find and regenerate.

The purpose of the find process is to peruse a propositional file in LTM and search for an image that is stored in the visual buffer and has been associated with an object's name. It will then run through a description of the foundation part that has been stored in the propositional file. Next, the find process will check, in an ordered sequence, the component parts that are comprised in the object so that they are reviewed.

With regard to the find process, Kosslyn (1980) has shown that when small digits are imagined next to an animal, they take longer to be seen than when they are imagined as being large. In another study, Kosslyn and Alper (1977) had subjects imagine two objects simultaneously. In half of the trials, one of the objects was imagined as smaller than the other, and in the other half, the two objects had the same size. In subsequent recall tasks, it was found that it took longer for subjects to recall the pair that contained the one small object. Kosslyn attributes this to the fact that the find process has to zoom in on the tiny object, which takes longer than it does merely to review the two similar objects.

The regenerate process reactivates parts of the image. To demonstrate this process, Kosslyn (1975) instructed subjects to image an animal next to a matrix containing either 4 or 16 cells. The subject was then given an animal part that either was or was not related to the imagined object. In either case, the subject was to mentally look for the part of the image on the animal. If the part 
could not be located on the animal, the subject was supposed to mentally assign it to the animal even if it was not actually a feature that the animal possessed. The subject was then to decide whether the part was appropriate to the animal. Kosslyn found that it took subjects longer to perform the task with the matrix that had the larger number of cells. He stipulated that as the number of parts increased, the time it took to refresh each part also increased. This was interpreted to mean that there would be more time between repeated activations and, thus a greater likelihood that parts would become faded. For this reason, parts of more complex images would require more time to generate.

What has not been shown is whether improvement in a task that is weighted in one particular process will lead to a generalized improvement in that particular process or ability. For instance, Kosslyn et al. (1984) asked whether improvement would be retained over time, and whether these improvements would generalize to improvement with new stimulus materials. In other words, would training on image maintenance subsequently improve overall performance, and would this be maintained over time?

To examine the find process, a mental rotation task described by Cooper $(1975,1976 a, 1976 b)$ was used. According to Kosslyn et al. (1984), such a task is weighted most heavily in the aforementioned process. They stipulate that the find process monitors the image as it is rotated, in this case when the image is at a specified orientation. The find process is also used to indicate the standard or mirror-reflected directions of the image.

Kosslyn et al. (1984) also found that the farther from $360^{\circ}$ that the image is rotated, the longer the speed of the rotation. They attribute this to taxing the find process. Therefore, if there is improvement in the find process with practice, rotation times should decrease. Additionally, mental rotation performance should improve with practice.

Another task that is weighted in the find process consists of the performing of geometric analogies (Novick \& Tversky, 1987). This task is related to spatial visualization abilities that involve image generation, transformation, and retention. To perform the task, subjects must manipulate images of geometric shapes to solve an analogy problem. There are seven possible transformations, including rotate, reflect, move, size, add, remove, and shading. Like mental rotation, performance on this task should also improve with practice.

In addition to a mental rotation task and a geometric analogies task, a line drawing memory task was used. This task was modeled after one used by Bower (1972) and Kosslyn (1983) to investigate the quantity of material subjects could hold in memory. This task requires subjects to imagine a series of 1 -in. lines varying in number of segments from two to six. The experimenter dictates a set of eight compass directions, including north, northwest, northeast, south, southwest, southeast, east, and west. The subjects must mentally connect the line segments to the preceding line segment on the basis of the direction dictated. After the presentation of the directions, the subjects must make a timed response as to whether the endpoint of the last line is above or below the origin of the starting line. The purpose of the timed response, as in the Kosslyn et al. (1984) study, was to enhance the possibility of subjects' using imagery to complete the task.

The aforementioned task was chosen because it has been established (Kosslyn et al., 1984) that the regenerate process is weighted most heavily in its execution. That is, the regenerate process is used by subjects to maintain the image of the line segments as the next direction is dictated. As each new direction is dictated, the previous image must be regenerated in order to add on the new line segment. When the directions are completed, the image must again be regenerated so that the correct response can be made and the configuration drawn.

As with the mental rotation and geometric analogies tasks, line drawing memory should improve with practice. In addition, Kosslyn et al. (1984) reported that as the number of line segments increased, accuracy decreased. They attribute this to taxing the regenerate process with increasing amounts of material. Therefore, if subjects are able to improve their accuracy scores with practice (as they did in the Kosslyn et al., 1984 study), this would indicate an improvement in the regenerate process.

In summary, the purpose of this study was to determine whether improvement in one process, due to practice in a task weighted in that process, would lead to a generalized improvement in the process and be demonstrated in other tasks also weighted in that process. That is, if individuals were to perform a task that was weighted in, say, the find process until improvement was shown, would this improvement generalize to improvement in another task that was also weighted in the find process and not generalize to improvement in a task weighted in another process such as regenerate? This was examined in Experiment 1.

\section{EXPERIMENT 1}

\section{Subjects \\ Method \\ Sixty undergraduate volunteers from introductory psychology classes at Cleveland State University participated in the experiment for extra course credit. None of the subjects had previously partic- ipated in experiments involving performance in imagery tasks.}

\section{Method and Procedure}

The volunteers were randomly assigned to one of four practice groups: a mental rotation (MR) practice, a line drawing (LD) practice, a time control $\left(C_{1}\right)$, or a distraction control $\left(C_{2}\right)$. In addition, all subjects were subsequently tested on a geometric analogies (GA) task taken from a study by Novick and Tversky (1987).

Mental rotation. The mental rotation practice was based on a paradigm described by Cooper $(1975,1976 \mathrm{a}, 1976 \mathrm{~b})$. Stimulus presentations of approximately $2.5 \mathrm{sec}$ each were made on an IBM PS 2, Model 30 computer with a monochrome display, using a program designed specifically for this study. The chosen stimuli were two-dimensional, ambiguous angular shapes. Such stimuli were selected because they were determined to be low in verbal association (Vanderplas \& Garvin, 1959), and because, as a result, recognition of stimuli would not play a role with regard to ease with which 
subjects could rotate them. In addition, three-dimensional shapes were avoided because they are generally considered more difficult for untrained subjects to rotate (see Parsons, 1987; Shepard \& Cooper, 1983; Shepard \& Farrell, 1985; Shepard \& Metzler, 1971). Each stimulus subtended an average of $3.1^{\circ}$ of visual angle.

Studies have also shown that there is a linear relationship between the number of points in the shape of a stimulus and its perceptual complexity (Attneave, 1957; Attneave \& Amoult, 1956; Vanderplas $\&$ Garvin, 1959). That is, during mental rotation tasks, as the number of points increases, the complexity of the shape logarithmically increases (Mumaw, Pellegrino, Kail, \& Carter, 1984). For this reason, all six shapes were six-pointed.

Each of the shapes was presented in five different orientations within a circle in the picture plane. These were at $60^{\circ}$ increments, starting with $60^{\circ}$, and including $120^{\circ}, 180^{\circ}, 240^{\circ}$, and $300^{\circ}$ positions. Because the $360^{\circ}$ position was used for the training session, it was not used as a test position. The stimuli were presented so that they faced in two directions. Because the shapes were ambiguous, each was first presented in one direction, which was arbitrarily determined to be its standard direction, and in the mirror image or reflected direction. The stimuli were rotated in a clockwise fashion. Figure 1 displays the six shapes used in the experiment, in both the standard and the mirror-reflected directions.

The subjects first participated in a training session in which they were familiarized with the orientations to be used during the experimental session. They were shown a series of flash cards, each containing a line starting at the center of a circle with an arrow indicating the degree of the rotation (illustrated as a composite in Figure 2). The subjects rehearsed these orientations until they could identify each without error.

It was also necessary for the subjects to be able to discriminate between standard and mirror-reflected directions prior to the experiment. As in the Cooper (1975) study, training included a printed visual display of the six experimental shapes in the standard and mirror-reflected directions and in the training orientation only. The subjects studied this display for $10 \mathrm{~min}$.

During the training session, the stimuli were also presented for $2.5 \mathrm{sec}$, in both the standard and the reflected directions. The subjects

$\Lambda$
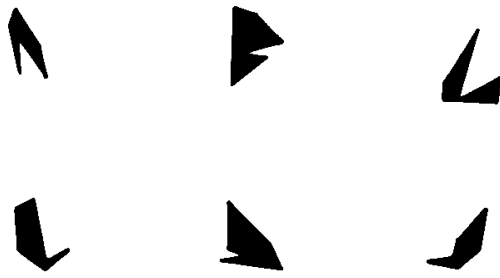

Standard
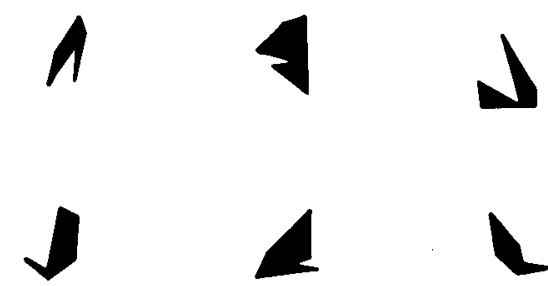

Mirror-reflected

Figure 1. Mental rotation shapes in the standard and mirrorreflected orientations.

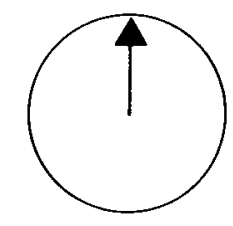

360

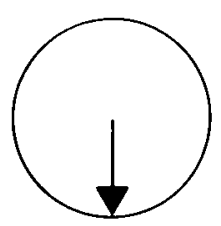

$180^{\circ}$

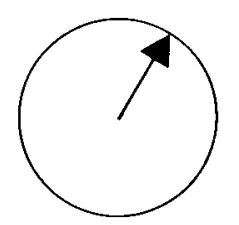

60

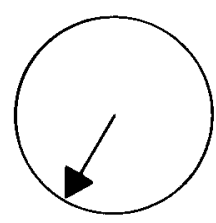

$\mathbf{2 4 0}$

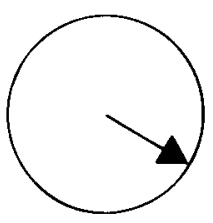

120

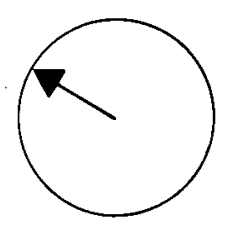

$300^{\circ}$
Figure 2. Flash card presentation orientations.

were asked to determine whether each orientation was in the standard or mirror-reflected position by pressing a key on the computer keyboard. The "S" key designated the standard response, and the " $M$ " key signified the mirror-reflected response. The training session consisted of presentation of each of the six shapes in both the standard and the mirror-reflected orientations for a total of 12 trials.

During the actual test session, the subjects were again shown the stimuli in 2.5-sec presentations. However, during the test sessions, the stimuli were presented in random fashion in all five orientations, excluding the training orientation. The subjects were asked to view the presentation and mentally rotate it in a clockwise fashion to the upright position, and to make a judgment as to whether it was in the standard or the mirror-reflected direction.

After making the responses, the subjects were required to press the keyboard space bar for the next stimulus presentation. This permitted them ample time between stimulus presentations. Reaction times (RTs) were measured in milliseconds from the onset of each stimulus presentation until the subjects pressed the response key.

Shepard and Cooper (1983) reported that subjects routinely rotate the shape to the upright position to distinguish a presented stimulus as either standard or mirror-reflected. However, subjects rotate the shape along the shortest path, unless specifically instructed otherwise. This means that stimuli presented before the $180^{\circ}$ position would be spontaneously rotated in a counterclockwise fashion to the upright. For this reason, as in the Kosslyn et al. (1984) and Cooper (1975) studies, the subjects were told to rotate the stimuli in a clockwise direction.

One experimental session included each of the six experimental shapes presented once in each of the five orientations. These were presented in both the standard and the mirror-reflected directions in random order, for a total of 60 presentations per session. At the end of each session, the computer displayed a readout of (1) the average amount of time (in milliseconds) to make the responses, (2) the number of correct responses, (3) the total number of standard responses, and (4) the number of mirror-reflected responses. The subjects were not provided feedback as to which individual responses were correct, so that any improvement made would be based on practice rather than on any learning through feedback.

The subjects continued the sessions until a ceiling on improvement was established. If the performance of the subjects decreased, they were reminded that the object was to maintain levels of performance or to improve, and they were instructed to try to restore their performance to prior levels.

Line drawing memory. Prior to the experimental session, the subjects were shown a series of flash cards of the eight compass 
directions used. Each card contained a 1-in. line randomly presented, and the subjects rehearsed until they were able to identify each direction without error. This had to be repeated from three to seven times for most of the subjects, because they varied in the degree to which they were comfortable identifying the directions. It also appeared that the diagonal directions--southwest, southeast, northwest, and northeast-were the most difficult to identify.

The subjects were then administered a practice session in which they were given pairs of two-direction and three-direction dictations, for a total of four practice dictations. They were asked to indicate whether the endpoint of the last line was above or below the origin of the starting line. They were also asked to draw the configuration.

After the training session, the experimenter dictated two- to sixline sequences in random order, for a total of five dictations per session. After the last dictation, the experimenter said, "endpoint." With this, the experimenter activated a Hunter Timer, Model $220 \mathrm{C}$. The timing procedure served as evidence to subjects that they were being timed; this served to increase the likelihood that imagery would be used to carry out the task. Upon hearing "endpoint," the subjects were required to indicate whether the endpoint of the last line was above or below the origin of the first line. The experimenter manually stopped the timer when the subjects made their responses. The subjects were then instructed to draw the configuration.

As in the mental rotation task, the subjects were not given feedback on each item. Nor were they instructed about which configurations were correctly drawn. Instead, they were shown the amount of improvement after several sessions. This was done to minimize the chances that either experimental group received more feedback on performance than the other. As was the case in the Kosslyn et al. (1984) study, the subjects showed no difficulty in performing the task. The tasks consisting of increasing numbers of line segments were in fact more difficult than the tasks with fewer line segments. To score the drawings, each individual line segment was scored in turn. If the line segment was in the correct direction from the endpoint of the preceding line segment, it was scored as correct.

As in the mental rotation task, subjects continued line drawing sessions until a ceiling on improvement was established. If the performance of subjects decreased, they were reminded that the object was to maintain levels of performance or to improve, and they were instructed to try to restore their performance to prior levels.

Time control. The third condition $\left(C_{1}\right)$ required the subjects to simply rest for a period of time approximately equal to that required to practice in the other groups. Because half of the geometric analogies were used for the pretest and half for the posttest, there was the possibility for subjects to show improvement on the posttest due to practice alone. To examine this possibility, $C_{1}$ was administered in only the pretest and the posttest geometric analogies tasks.

Distraction control. In the fourth condition $\left(C_{2}\right)$, the subjects were administered a series of timed cognitive and visual tasks: the Closure Speed Test (Thurstone \& Jeffrey, 1966), Copying Test Cf-3 from the Educational Testing Service, and Hidden Figures Test Cf-1 from the Educational Testing Service. These tasks were not loaded on either find or regenerate, which the subjects were required to perform between the pre- and posttests. This group was used because the subjects in $\mathbf{C}_{1}$ did not receive an experimental treatment between the pretest and posttest geometric analogies tasks. Because there is a possibility that performing any task might influence the outcome of the posttest, this second control was used.

Geometric analogies. The geometric analogies task is a paperand-pencil instrument that requires subjects to perform two transformations on a geometric figure. The task requires the subjects to view the first three parts of a geometric analogy problem on one page and to select a solution from five possible choices on another page. The fact that the subjects are not permitted to flip back and forth from page to page makes it necessary for them to form an image of the solution and to retain that image until they identify it on the response page.
The test was divided in half for the pre- and posttest sessions. Both sessions consisted of 2 of each of the 21 problem types, for a total of 42 problems on each. The tests were randomized in such a way that both sets were administered at the pre- and posttest sessions. However, no 2 subjects received the same pre- and posttests.

Prior to each test session, the subjects were instructed about the seven transformations via flash card examples of each. As in the Novick and Tversky (1987) study, the subjects were instructed to identify the transformations used. They were further instructed to perform these transformations to obtain an image of the solution. When the image was obtained, they were to turn the page and select the correct solution. They were instructed not to turn back to the preceding page, and if the image was lost or unobtainable, to make the best possible guess. The number of correct responses was the dependent measure.

It was necessary to observe whether improvement was made from the pre- to the posttest sessions on the geometric analogies task. It was therefore decided that a 10 -min time limit would be added to make the task more difficult.

Other tests. In addition, all subjects were administered the Gordon (1949) Test of Visual Imagery Control (TVIC), as well as the Vividness of Visual Imagery Questionnaire (VVIQ; Marks, 1973, 1989). These self-report questionnaires purport to measure an individual's ability to control as well as to manipulate a mental image. Many studies (e.g., Perry, 1973; Sutcliffe, Perry, \& Sheehan, 1970; Wallace, $1990 \mathrm{a}, 1990 \mathrm{~b})$ have reported a positive relationship between vividness of imagery as assessed on a standardized questionnaire and performance on tasks involving imagery. Therefore, one would expect that such a relationship should be present here.

\section{Results}

On the basis of the results from the Kosslyn et al. (1984) study, it was expected that subjects would improve with practice on both the mental rotation task and the line drawing memory task. Because a trials-by-criterion paradigm was used for both tasks, the first and best trials per task were considered. The first trial in the experimental session was recorded as a baseline measure of performance for subjects. The trial containing the subjects' best score, but not necessarily the last trial, was recorded as an index of the amount of improvement subjects had made.

After an analysis of variance (ANOVA) was conducted to determine whether a significant trials effect was present, Newman-Keuls analyses between first and best trials were performed to determine whether improvement made in these tasks was significant.

In the mental rotation task, both speed and accuracy were the dependent measures. Newman-Keuls analyses between first and best trials were performed for the number of correct mental rotations and for the time (in milliseconds) required to complete each experimental session.

On the accuracy trials, the number of correct mental rotations was significantly greater $(M=44.34, S D=$ $5.98)$ on best trials than on first trials $(M=36.56, S D=$ 5.34) (Newman-Keuls, $p<.05$ ). Likewise, the best trials $(M=195 \mathrm{msec}, S D=111)$ were significantly faster than on first trials $(M=392 \mathrm{msec}, S D=203$ ) (NewmanKeuls, $p<.01$ ).

The dependent measure in the line drawing memory task was the number of lines drawn in the correct direction. Results for this task were measured by starting with the 


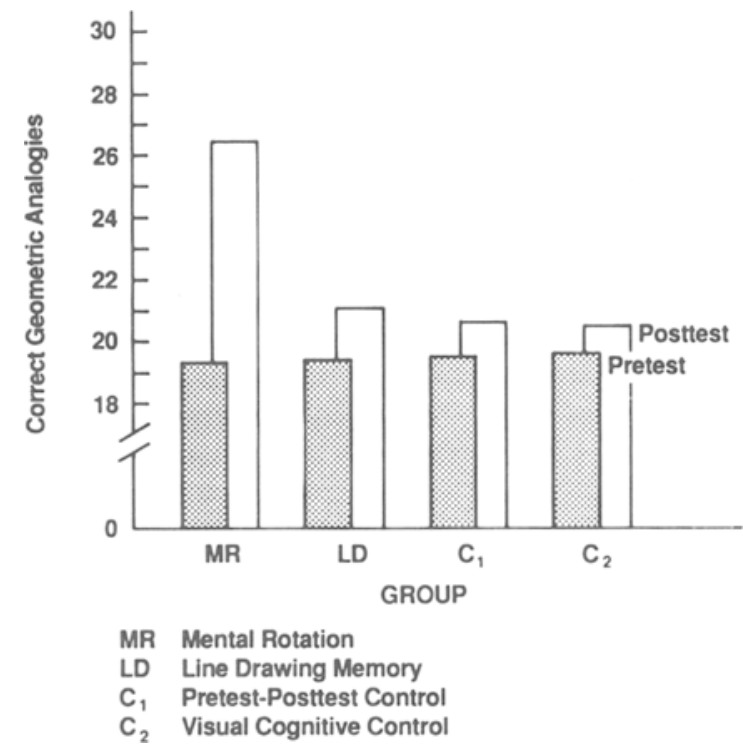

Figure 3. Pretest and posttest performance (number correct) on the geometric analogies task, as a function of group assignment.

three-line dictation and continuing through the six-line dictation. Two-line dictations were omitted from the analyses because all but 1 subject correctly drew the configuration on the first trial. Following a significant trials effect, Newman-Keuls analyses were again performed on first and best trials for number of lines correctly drawn for each of the three- through six-line dictations as well as for overall trials.

The results showed that best trials for all three- through six-line dictations produced significantly more lines drawn in the correct direction than did first trials (all NewmanKeuls, $p<.05$ ). The number of correct lines drawn were as follows: three-line dictation first trials $(M=1.02$,
$S D=0.48)$, best trials $(M=3.00, S D=0.00)$; fourline dictation first trials $(M=1.82, S D=1.08)$, best trials $(M=3.92, S D=0.35)$; five-line dictation first trials $(M=1.68, S D=0.86)$, best trials $(M=4.47$, $S D=1.55)$; and six-line dictation first trials $(M=1.49$, $S D=0.76)$, best trials $(M=5.24, S D=1.05)$.

In summary, the subjects in the mental rotation group showed significant improvement in both speed and accuracy with practice on the mental rotation task. Likewise, the subjects in the line drawing memory group showed significant improvement in number of lines correctly drawn with practice on the three-through six-line dictations. This replicates the results reported by Kosslyn et al. (1984), who demonstrated that subjects showed significant improvement with practice on these two tasks.

A 4 (group) $\times 2$ (pretest vs. posttest) mixed design ANOVA was performed on the number of correct geometric analogies obtained. The results showed a significant interaction $[F(3,56)=19.37, p<.001$; see Figure 3]. The mental rotation group outperformed the three other groups on the geometric analogies posttest (NewmanKeuls, $p<.05$ ). It also outperformed all four groups on the geometric analogies pretests. In addition, NewmanKeuls analyses indicated that pretest performance was not significantly different among any of the four groups. Similar results were obtained when the analysis was repeated on the total number of analogies completed.

Correlational analyses were performed (see Table 1) to determine whether the geometric analogies and mental rotation tasks were weighted by the same process. Results showed significant correlations between scores on the pregeometric analogies task and both first trial scores $[r(13)=.54, p<.05]$ and best trial scores $[r(13)=$ $.52, p<.05$ ] on the mental rotation tasks. Significant correlations were also obtained between scores on the postgeometric analogies task and both first trial scores $[r(13)=.52, p<.05]$ and best trial scores $[r(13)=$ $.55, p<.05]$ on the mental rotation task.

Table 1

\begin{tabular}{|c|c|c|c|c|c|c|c|c|c|c|c|c|c|c|c|}
\hline & \multirow{3}{*}{\multicolumn{2}{|c|}{$\begin{array}{l}\text { Geometric } \\
\text { Analogies }\end{array}$}} & \multirow{2}{*}{\multicolumn{3}{|c|}{$\begin{array}{c}\text { Mental } \\
\text { Rotations }\end{array}$}} & \multicolumn{8}{|c|}{ Line Dictations } & \multirow[b]{4}{*}{$\frac{\text { TVIC }}{\text { (14) }}$} & \multirow[b]{4}{*}{$\frac{\text { VVIQ }}{(15)}$} \\
\hline & & & & & & \multicolumn{2}{|c|}{ Three-Line } & \multicolumn{2}{|c|}{ Four-Line } & \multicolumn{2}{|c|}{ Five-Line } & \multicolumn{2}{|c|}{ Six-Line } & & \\
\hline & & & First & Best & & First & Best & First & Best & First & Best & First & Best & & \\
\hline & $\frac{\text { Pretest }}{(1)}$ & $\frac{\text { Posttest }}{(2)}$ & $\frac{\text { Trial }}{(3)}$ & $\frac{\text { Trial }}{(4)}$ & $\frac{\text { Speed }}{(5)}$ & $\frac{\text { Trial }}{(6)}$ & $\frac{\text { Trial }}{(7)}$ & $\frac{\text { Trial }}{(8)}$ & $\frac{\text { Trial }}{(9)}$ & $\frac{\text { Trial }}{(10)}$ & $\frac{\text { Trial }}{(11)}$ & $\frac{\text { Trial }}{(12)}$ & $\frac{\text { Trial }}{(13)}$ & & \\
\hline$\overline{(1)}$ & 1.00 & & & & & & & & & & & & & & \\
\hline (2) & .39 & 1.00 & & & & & & & & & & & & & \\
\hline (3) & $.54^{*}$ & $.52^{*}$ & 1.00 & & & & & & & & & & & & \\
\hline (4) & $.52^{*}$ & $.55^{*}$ & .50 & 1.00 & & & & & & & & & & & \\
\hline (5) & .49 & .43 & .34 & .37 & 1.00 & & & & & & & & & & \\
\hline (6) & .22 & .30 & .24 & .11 & .24 & 1.00 & & & & & & & & & \\
\hline (7) & .24 & .21 & .29 & .24 & .21 & .38 & 1.00 & & & & & & & & \\
\hline (8) & .18 & .18 & .14 & .09 & .19 & .46 & .37 & 1.00 & & & & & & & \\
\hline (9) & .11 & .14 & .21 & .14 & .08 & .31 & $.68 \ddagger$ & .34 & 1.00 & & & & & & \\
\hline (10) & .14 & .22 & .36 & .28 & .09 & .29 & .41 & .49 & .36 & 1.00 & & & & & \\
\hline (11) & .15 & .18 & .24 & .28 & .22 & .44 & .30 & .46 & .41 & .45 & 1.00 & & & & \\
\hline (12) & .20 & .11 & .08 & .33 & .19 & .27 & .41 & .47 & .39 & .41 & .38 & 1.00 & & & \\
\hline (13) & .09 & .13 & .22 & .19 & .30 & $.64 t$ & .39 & .30 & .46 & .39 & .46 & .31 & 1.00 & & \\
\hline (14) & $.57^{*}$ & $.64 \dagger$ & $.58 *$ & $.57^{*}$ & $.68 \ddagger$ & .24 & .13 & .06 & .09 & .11 & .06 & .21 & .14 & 1.00 & \\
\hline (15) & .16 & .24 & .09 & .11 & .06 & $.54 *$ & $.61 \dagger$ & $.52^{*}$ & $.57^{*}$ & $.53 *$ & $.55^{*}$ & $.52^{*}$ & $.52^{*}$ & .16 & 1.00 \\
\hline
\end{tabular}

${ }^{*} p<.05 . \quad \dagger p<.02 . \quad \ddagger p<.01$. 
Significant correlations were also found between TVIC scores and first trial $[r(13)=.58, p<.05]$ and best trial $[r(13)=.57, p<.05]$ performance on the mental rotation task. The TVIC also correlated significantly with speed of rotation $[r(13)=.68, p<.01]$. The VVIQ did not correlate with any of the aforementioned. However, the VVIQ did correlate with performance on the line drawing memory task for three-, four-, five-, and six-line dictations (see Table 1). Also, for the three-, four-, five-, and six-line dictations in the line drawing memory task, neither first trial nor best trial scores correlated with either pretest or posttest geometric analogies scores. Finally, VVIQ scores and TVIC scores were not significantly correlated.

\section{Discussion}

On the basis of the results of previous experiments done by Kosslyn and his colleagues, we predicted that subjects who performed a mental rotation task or a line drawing memory task would show improvement in performance with practice. The results from Experiment 1 support these predictions.

We also predicted that improvement would generalize to improvement on another task weighted in the same process. Indeed, results showed that the mental rotation group improved both in accuracy and in number completed from the pretest to the posttest for geometric analogies, another task weighted in the find process. Such improvement from pretest to posttest was significant only when both tasks were weighted by the same process, presumably the find process.

The generalization of improvement might reflect the fact that both mental rotation and the geometric analogies task require image transformation, including mental rotation specifically. However, even if this is the case; the results of Experiment 1 are consistent with Kosslyn's (1980, 1983) theory that postulates find and regenerate processes. This is bolstered by the presence of a significant correlation between scores on the geometric analogies task and scores on accuracy for the mental rotation task. This correlation is consistent with the view that these two tasks share some corresponding process. Because both tasks were theoretically weighted in the find process, it is likely that this is the process that was shared by the two tasks.

Improvement on the line drawing memory task, however, did not significantly affect performance on the geometric analogies task. This suggests that this task does not share a similar process with the geometric analogies task or the mental rotation task. In other words, as Kosslyn and his colleagues have stipulated, imaging ability comprises several independent processes. In the case of line drawing, Kosslyn and his colleagues have suggested that the process most likely involved is regenerate. Thus, at least for the manipulations considered in Experiment 1, find and regenerate appear to be separate imagery processes.

If performance on mental rotation generalizes to performance on a geometric analogies test, might the reverse also be true? In Experiment 2, we examined the possibility of generalizing improvement from the geometric analogies to the mental rotation task, the reverse direction of that tested in Experiment 1.

\section{EXPERIMENT 2}

\section{Method}

\section{Subjects}

Sixty undergraduates from introductory psychology classes participated for extra course credit. None of the subjects had participated in Experiment 1 or in other experiments involving performance in an imagery task.

\section{Procedure}

The procedure was similar to that in Experiment 1. However, one group of subjects was given practice on the geometric analogies task instead of a mental rotation task. Because performance on mental rotation ability was to be assessed as a function of group assignment (see Experiment 1 for descriptions), half of the rotation combinations (randomly selected) previously described were used to establish pretest performance; half served as a posttest. In addition, as in Experiment 1, all subjects were administered the TVIC and the VVIQ (random order assignment for testing).

\section{Results}

As in Experiment 1, performance improved significantly with practice for both the geometric and the line drawing memory task for three- through six-line dictations (all Newman-Keuls comparisons yielded $p$ values of .05 or better).

In addition, two dependent measures were examined: correct number of mental rotations and speed with which mental rotations were performed. As shown in Figure 4, group assignment interacted with pretest/posttest performance for correct mental rotation $[F(3,56)=3.41$, $p<.01]$ because the geometric analogies group had

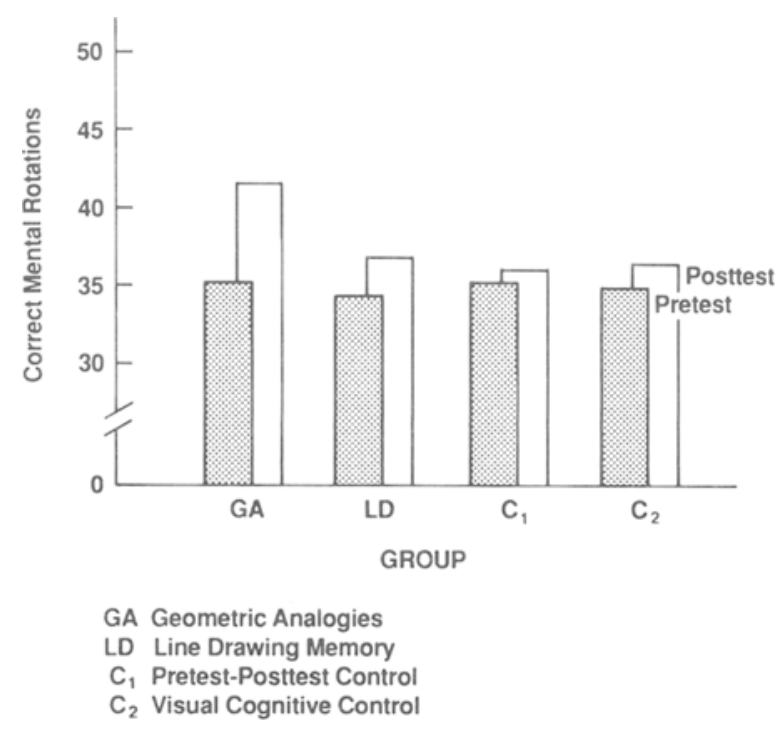

Figure 4. Mean correct mental rotations during pretest and posttest performance, as a function of group assignment. 


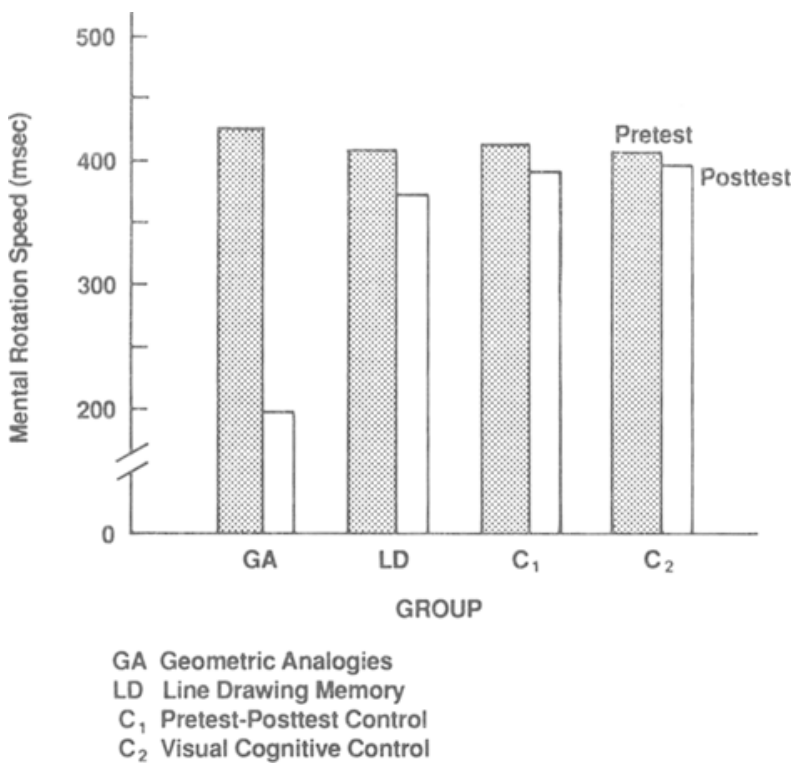

Figure 5. Mental rotation speed (in milliseconds) during pretest and posttest performance, as a function of group assignment.

higher posttest scores than the pretest performance for each of the four groups (Newman-Keuls, $p<.05$ ). In addition, Newman-Keuls analyses indicated that pretest performance did not significantly differ among any of the four groups.

Analyses of the speed of mental rotations yielded similar results (see Figure 5). The geometric analogies group correctly performed an average of 41.3 posttest mental rotations $(S D=7.5)$; their pretest rotations came to an average of $35.2(S D=5.8)$. The posttest performance of this group was significantly better than the posttest results of the other three groups and than the pretest performance for each of the four groups (Newman-Keuls, $p<.01)$. Pretest performance did not differ significantly for the groups.

Correlational analyses again were performed (see Table 2) to determine whether the mental rotation and geometric analogies tasks were weighted in the same process. The number of correct mental rotations produced in the pretest correlated significantly with both the first trial geometric analogies scores $[r(13)=.55, p<.05]$ and with the best trial geometric analogies scores $[r(13)=$ $.61, p<.02$ ]. Correlations between the number of correct mental rotations produced in the posttest with both first and best trial geometric analogies performance were also significant $[r(13)=.68, p<.02$, and $r(13)=.53$, $p<.05$, respectively]. These results are similar to those reported in Experiment 1.

For the three-, four-, five-, and six-line dictations in the line drawing memory task, neither first trial nor best trial scores correlated with either pretest or posttest mental rotation performance for either of the dependent measures.

When TVIC and VVIQ scores were examined as a function of the various dependent measures, significant correlations were found for the same measures as were described in Experiment 1 (see Table 2). Finally, VVIQ scores and TVIC scores did not correlate with each other.

\section{Discussion}

Experiment 2 provides further evidence that performance on one find process task generalizes to performance on another find process task. Although the two tasks were identical to those used in Experiment 1, the results here illustrate that generalizability is bidirectional. That is, there appeared to be nothing unique about generalizing from performance on a mental rotation task to performance on a geometric analogies task because generalization here occurred from geometric analogies to mental rotation.

Table 2

\begin{tabular}{|c|c|c|c|c|c|c|c|c|c|c|c|c|c|c|c|}
\hline & \multirow{2}{*}{\multicolumn{2}{|c|}{$\begin{array}{l}\text { Geometric } \\
\text { Analogies }\end{array}$}} & \multirow{3}{*}{\multicolumn{3}{|c|}{$\begin{array}{c}\text { Mental } \\
\text { Rotations }\end{array}$}} & \multicolumn{8}{|c|}{ Line Dictations } & \multirow{5}{*}{$\frac{\text { TVIC }}{(14)}$} & \multirow{5}{*}{$\frac{\text { VVIQ }}{(15)}$} \\
\hline & & & & & & \multicolumn{2}{|c|}{ Three-Line } & \multicolumn{2}{|c|}{ Four-Line } & \multicolumn{2}{|c|}{ Five-Line } & \multicolumn{2}{|c|}{ Six-Line } & & \\
\hline & First & $\overline{\text { Best }}$ & & & & First & $\overline{\text { Best }}$ & First & Best & First & Best & First & Best & & \\
\hline & Trial & Trial & Pretest & Posttest & Speed & Trial & Trial & Trial & Trial & Trial & Trial & Trial & Trial & & \\
\hline & (1) & (2) & (3) & (4) & (5) & $\overline{(6)}$ & $\overline{(7)}$ & $(8)$ & (9) & $\overline{(10)}$ & $\overline{(11)}$ & $\overline{(12)}$ & $\overline{(13)}$ & & \\
\hline (1) & 1.00 & & & & & & & & & & & & & & \\
\hline (2) & .42 & 1.00 & & & & & & & & & & & & & \\
\hline (3) & $.55^{*}$ & $.61 \dagger$ & 1.00 & & & & & & & & & & & & \\
\hline (4) & $.68 \ddagger$ & $.53^{*}$ & .51 & 1.00 & & & & & & & & & & & \\
\hline (5) & .39 & .36 & .39 & .41 & 1.00 & & & & & & & & & & \\
\hline (6) & .24 & .18 & .16 & .20 & .21 & 1.00 & & & & & & & & & \\
\hline (7) & .30 & .24 & .22 & .15 & .19 & .27 & 1.00 & & & & & & & & \\
\hline (8) & .22 & .19 & .35 & .04 & .17 & .39 & .28 & 1.00 & & & & & & & \\
\hline (9) & .17 & .24 & .07 & .21 & .34 & .42 & .39 & .49 & 1.00 & & & & & & \\
\hline (10) & .34 & .28 & .19 & .16 & $.52 *$ & 38 & .42 & .27 & .40 & 1.00 & & & & & \\
\hline (11) & .26 & .21 & .24 & .30 & .31 & .40 & $.53^{*}$ & .29 & .41 & .36 & 1.00 & & & & \\
\hline (12) & .31 & .17 & .04 & .17 & .12 & .31 & .42 & .41 & .38 & .29 & .44 & 1.00 & & & \\
\hline (13) & .26 & .15 & .17 & .29 & .24 & .36 & .39 & .50 & $.54^{*}$ & .39 & .42 & .41 & 1.00 & & \\
\hline (14) & $.65+$ & $.67 \dagger$ & $.76 \S$ & $.78 \$$ & .22 & .09 & .07 & 19 & 21 & .15 & 19 & 22 & 19 & 1.00 & \\
\hline (15) & .24 & .22 & .19 & $.17^{\circ}$ & .03 & $.72 \S$ & $.75 \S$ & $.69 \ddagger$ & $.66+$ & $.70 \ddagger$ & $.65 \dagger$ & $.71 \S$ & $.61 \dagger$ & .21 & 1.00 \\
\hline
\end{tabular}

${ }^{*} p<.05 . \quad \ddagger p<.02 . \quad \ddagger p<.01 . \quad \S p<.001$. 
The first two experiments were concerned only with the generalizability of tasks within the find process. However, Kosslyn (1983) has described several processes. If his theory is sufficiently robust concerning generalization within a process, phenomena such as those demonstrated in Experiments 1 and 2 should also exist within other processes. Because the regenerate process was considered in the previous experiments as a process independent from the find process, in Experiment 3 we examined the possibility of generalizability within this process. It was predicted that performance on a task loaded in the regenerate process should generalize to performance on another task loaded on this process.

\section{EXPERIMENT 3}

\section{Method}

\section{Subjects}

Sixty undergraduates from introductory psychology classes participated for extra course credit. None of the subjects had participated in either of the previous experiments or in other experiments involving performance in an imagery task.

\section{Procedure}

The procedure was similar to that in Experiment 1, except that a variation of Kosslyn's (1975) animal imagery task was substituted for line drawing memory as a group assignment. Kosslyn (1983) has described this task as one that loads on his regenerate process.

The animal imagery task required subjects to image an animal next to a matrix. In the present experiment, the subject was instructed to image the animal as superimposed upon a 16-cell matrix. The subject would then be given an animal part that was or was not related to the imagined object and would be asked to locate it. If the part could not be located, the subject was asked to mentally still assign it to the animal even if it was not actually a feature that the animal possessed. The subject was then to decide whether the part was appropriate to the animal.

The dependent measures were the time required for subjects to perform the task and the accuracy with which they located parts. The latter measure could be ascertained because subjects called off numbers that corresponded to loci on the matrix for the various body parts. Cell values were assigned by asking the subjects to number each, starting from the top left and moving to the right and then moving to the second row, and so on. The experimenter could then compare for accuracy on a template prepared with an animal superimposed on a 16-cell matrix. These templates were not shared with the subjects. As in Experiment 1 for use with the line drawing memory task, a Hunter timer recorded speed information. The presence of the timer served as evidence to subjects that time was an important element of the experiment.

Because the primary reason for this experiment was to determine whether performance on one regenerate task would generalize to performance on another such task, the line drawing memory task (previously described) was also employed here. For the purpose of this experiment, 10 each of three-, four-, five-, and six-line dictations were used. Five of each (randomly selected) were used to determine baseline performance. The remaining dictations were used to assess posttest performance as a function of group assignment.

As in the previous experiments, subjects were randomly assigned to one of four groups (MR, AI, $C_{1}$, or $C_{2}$, for this experiment). And subjects were treated identically to those in Experiment 1 for the $M R, C_{1}$, and $C_{2}$ conditions.

In the AI condition, the subjects first participated in a training session to familiarize them with the process that would be required. They were each asked to imagine a cat and a horse on a 16-cell matrix (randomly ordered). Using their imagined and numbered cell matrix, the experimenter asked the subjects to locate three parts (one at a time) for each animal. The subjects were then asked to find the part on their imagined matrix and to indicate its numerical position. The subjects were permitted to practice on these samples until they indicated that they were ready for other stimuli (averaged practice time $=3.5 \mathrm{~min}$ ).

Following practice, the animal imagery subjects were asked to imagine 10 additional animals (chicken, dog, duck, elephant, fox, giraffe, gorilla, pig, skunk, and squirrel). As with the practice samples, the subjects were asked to locate three parts (one at a time) per animal. The order of presentation was random. As with the practice animals, they were to indicate the position of a body part for each stimulus.

\section{Results}

As in Experiment 1 with the mental rotation task, both speed and accuracy improved significantly with practice. The number of correct mental rotations was greater on best trials $(M=41.18, S D=6.05)$ than on first trials $(M=34.88, S D=5.94)$ (Newman-Keuls, $p<.05)$. Also, best trials $(M=212 \mathrm{msec}, S D=94)$ were significantly faster than first trials $(M=418 \mathrm{msec}, S D=128)$ (Newman-Keuls, $p<.01$ ).

On the animal imagery task, accuracy in locating body parts significantly improved in best trials relative to first trials. When averaged over different animals and different animal parts, best trials produced 22.13 correct placements $(S D=4.89)$ compared with 15.69 correct placements $(S D=3.66)$ for first trials (Newman-Keuls, $p<.05)$. Also, best trials produced faster locating of parts $(1.67 \mathrm{sec}, S D=1.04)$ than did first trials $(4.86 \mathrm{sec}$, $S D=1.94)$ (Newman-Keuls, $p<.001)$.

A significant group $\times$ pretest/posttest interaction was produced for number of lines correctly drawn when averaged over three-, four-, five-, and six-line dictations $[F(3,56)=5.09, p<.01$; see Figure 6$]$. This interaction occurred because the animal imagery group performed significantly better on the line drawing memory posttest (Newman-Keuls, $p<.05$ ) than did the other

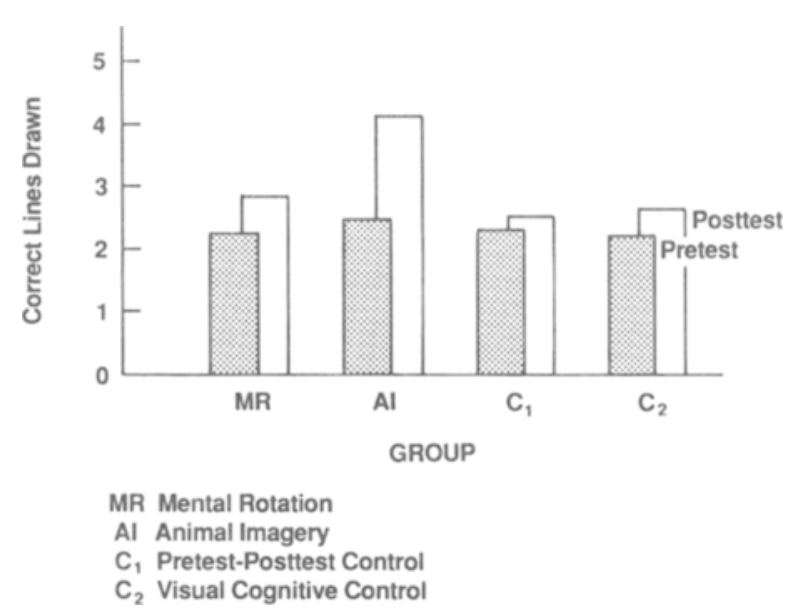

Figure 6. Line drawing memory averaged over types of dictation during pretest and posttest performance, as a function of group assignment. 
Table 3

\begin{tabular}{|c|c|c|c|c|c|c|c|c|}
\hline & \multirow{3}{*}{$\begin{array}{c}\text { Line } \\
\text { Drawing } \\
\text { Accuracy } \\
\end{array}$} & \multicolumn{3}{|c|}{ Mental Rotation } & \multirow{2}{*}{\multicolumn{2}{|c|}{ Animal Imagery }} & \multirow[b]{3}{*}{ TVIC } & \multirow[b]{3}{*}{ VVIQ } \\
\hline & & \multirow{2}{*}{$\begin{array}{l}\text { First } \\
\text { Trial }\end{array}$} & \multirow{2}{*}{$\begin{array}{l}\text { Best } \\
\text { Trial } \\
\end{array}$} & \multirow[b]{2}{*}{ Speed } & & & & \\
\hline & & & & & Speed & Accuracy & & \\
\hline & (1) & (2) & (3) & (4) & (5) & (6) & (7) & (8) \\
\hline (1) & 1.00 & & & & & & & \\
\hline (2) & .24 & 1.00 & & & & & & \\
\hline (3) & .19 & .41 & 1.00 & & & & & \\
\hline (4) & .30 & .38 & .34 & 1.00 & & & & \\
\hline (5) & $.54^{*}$ & .15 & .19 & .27 & 1.00 & & & \\
\hline (6) & $.66 t$ & .24 & .30 & .21 & .36 & 1.00 & & \\
\hline (7) & .17 & $.70 \ddagger$ & $.52^{*}$ & $.59 *$ & .24 & .20 & 1.00 & \\
\hline (8) & $.61 t$ & .21 & .14 & .08 & $.55^{*}$ & $.61 \dagger$ & .18 & 1.00 \\
\hline
\end{tabular}

three groups and better than the pretest performance for each of the four groups. In addition, Newman-Keuls analyses indicated that pretest performance was not significantly different among any of the four groups.

A correlational analysis was performed (see Table 3) to determine whether the line drawing memory task and the animal imagery task were weighted in the same process. The results showed a significant correlation between the average number of correct line drawings and the accuracy with which subjects identified animal parts in imagery $[r(13)=.66, p<.05]$. The average number of correct line drawings also correlated with the speed with which subjects located animal parts $[r(13)=.54$, $p<.05$ ]. Correlations between performances of subjects in the mental rotation group with line drawing accuracy were not significant.

When TVIC and VVIQ scores were compared with performance on the various tasks in Experiment 3 (see Table 3), the former correlated with mental rotation performance for accuracy on both first trials $[r(13)=.70$, $p<.01]$ and best trials $[r(13)=.52, p<.05]$. The TVIC also correlated with speed of rotation $[r(13)=.59$, $p<.05$ ]. As in Experiment 1, the VVIQ did not correlate with any of the aforementioned. However, the VVIQ did correlate with performance on animal imagery, both for accuracy of report $[r(13)=.61, p<.02]$ and for speed of locating parts $[r(13)=.55, p<.05]$. Finally, as in Experiment 1, VVIQ scores and TVIC scores did not significantly correlate.

\section{Discussion}

The results of Experiment 3 follow the pattern of the results in the previous two experiments. Subjects' performance on a task weighted in one process generalized to performance on another task weighted by the same process. Thus, performance on animal imagery that is weighted in the regenerate process generalizes to superior performance in line drawing memory, another task weighted in Kosslyn's (1983) regenerate process.

The results reported in Experiments 1 and 2 appear not to be unique to tasks weighted only in the find process or to tasks that manipulate only image transformations. As is clear in Experiment 3, generalizability is just as robust when one considers the regenerate process.

\section{GENERAL DISCUSSION}

From the results of the experiments reported, find and regenerate appear to be separate and independent imagery processes. This is confirmed by the results of the three experiments as well as by the correlations between the various results and scores on the TVIC and the VVIQ.

The aforementioned findings have obvious implications for Kosslyn's (1975, 1980, 1983) model. He predicted the independence of processes as has been demonstrated here, and our findings are thus consistent with his predictions. However, as was mentioned in the section on Experiment 1, transfer could simply be explained in terms of practice. That is, if one practices mental rotation in a paradigm such as that used in Experiment 1, transfer of learning to another paradigm that relies on mental rotation (geometric analogies) might be expected. Thus, mental rotation transfers to mental rotation. Such an explanation could also be offered for Experiment 2. Similarly, the skill involved in performing the tasks described in Experiment 3, adding information to an existing mental image, might also be explained as a result of transfer of learning. In essence, transfer takes place because of practice. Thus, although our results are consistent with predictions made by Kosslyn's model, there may be a simpler explanation for our findings.

Future investigators of imagery ability should be aware of the apparent independence of imagery processes, and they should remember that different imagery questionnaires seem to predict different processes like those postulated in Kosslyn's $(1975,1980,1983)$ model. Investigators should recognize that the choice of the test may affect the results produced.

In the present study we have reported some evidence to support the independence of some imagery processes, but we did not attempt to exhaustively investigate the independence of all of the various imagery processes delineated by Kosslyn and his colleagues. Prudence suggests further studies to consider the independence of processes not considered here.

Interestingly, independence of the imagery processes stipulated by Kosslyn and his colleagues is supported by the correlational results reported with regard to the TVIC and the VVIQ. The TVIC is considered to be an imagery 
assessment tool that measures an individual's ability to control as well as to manipulate a mental image. With regard to performance on the mental rotation task and on the geometric analogies task, subjects are probably exhibiting their ability to control and to manipulate a mental image. Given the significant correlations reported between performance on a mental rotation task, performance on a geometric analogies task, and performance on the TVIC, this ability to control and to manipulate is likely a part of the find process as described by Kosslyn and his colleagues.

On the other hand, the VVIQ purportedly measures an individual's imagery vividness. Our results suggest that imagery vividness is not important to either a mental rotation or a geometric analogies task. It is important, however, in a line drawing memory task. Subjects who demonstrate vivid imaging ability as assessed by the VVIQ appear to perform best.

\section{REFERENCES}

Attneave, F. (1957). Physical determinants of the judged complexity of shapes. Journal of Experimental Psychology, 53, 221-227.

Attneave, F., ARnoult, M. D. (1956). The quantitative study of shape and pattern perception. Psychological Bulletin, 53, 452-471.

Bower, G. H. (1972). Mental imagery and associative learning. In L. Gregg (Ed.), Cognition in learning and memory (pp. 51-88). New York: Wiley.

COOPER, L. A. (1975). Mental rotation of random two-dimensional shapes. Cognitive Psychology, 7, 20-43.

COOPER, L. A. (1976a). Demonstration of a mental analog of an external rotation. Perception \& Psychophysics, 19, 296-302.

COOPER, L. A. (1976b). Individual differences in visual comparison processes. Perception \& Psychophysics, 19, 433-444.

GorDon, R. (1949). An investigation into some of the factors that favor the formation of stereotyped images. British Journal of Psychology, 39, 156-167.

KossLYN, S. M. (1975). Information representation in visual images. Cognitive Psychology, 7, 341-370.

Kosslyn, S. M. (1980). Image and mind. Cambridge, MA: Harvard University Press.

KossLyn, S. M. (1983). Ghosts in the mind's machine: Creating and using images in the brain. New York: Norton.

Kosslyn, S. M., \& ALPER, S. N. (1977). On the pictorial properties of visual images: Effects of image size on memory for words. Canadian Journal of Psychology, 31, 32-40.

Kosslyn, S. M., BrunN, J., Cave, K. R., \& Wallach, R. W. (1984). Individual differences in mental imagery ability: A computational analysis. Cognition, 18, 195-243.

Kosslyn, S. M., Reiser, B. J., Farah, M. J., Fllegel, S. L. (1983). Generating visual images. Joumal of Experimental Psychology: General, 112, 278-303.

MARKS, D. F. (1973). Visual imagery differences in the recall of pictures. British Journal of Psychology, 64, 17-24.

MARKS, D. F. (1989). Construct validity of the Vividness of Visual Imagery Questionnaire. Perceptual \& Motor Skills, 69, 459-465.

Mumaw, R. J., Pellegrino, J. W., Kail, R. V., Jr., Carter, P. (1984). Different slopes for different folks: Process analysis of spatial aptitude. Memory \& Cognition, 12, 515-521.

Novick, L. R., \& TVERSKY, B. (1987). Cognitive constraints on ordering operations: The case of geometric analogies. Journal of Experimental Psychology: General, 116, 50-67.

PARSONS, L. M. (1987). Visual discrimination of abstract mirrorreflected three-dimensional objects at many orientations. Perception \& Psychophysics, 42, 49-59.

PerRy, C. W. (1973). Imagery, fantasy, and hypnotic susceptibility. Journal of Personality \& Social Psychology, 26, 217-221.

ShePARD, R. N., \& COOPER, L. A. (1983). Mental images and their transformations. Cambridge, MA: MIT Press.

SHEPARD, R. N., FARRELL, J. E. (1985). Representation of the orientation of shapes. Acta Psychologica, 59, 103-121.

ShePARd, R. N., \& MeTzLeR, J. (1971). Mental rotation of threedimensional objects. Science, 171, 701-703.

Sutcliffe, J. P., Perry, C. W., \& Sheehan, P. A. (1970). Relation of some aspects of imagery and fantasy to hypnotic susceptibility. Journal of Abnormal Psychology, 76, 279-287.

Thurstone, L. L., Jefrrey, T. E. (1966). Closure Speed Test administration manual. Chicago: University of Chicago, Industrial Relations Center.

Vanderplas, J. M., \& Garvin, E. A. (1959). Complexity, association value, and practice as factors in shape recognition following pairedassociate training. Journal of Experimental Psychology, 57, 155-163.

WALLACE, B. (1990a). Hypnotic susceptibility and the modification of cognitive search strategies. International Journal of Clinical \& Experimental Hypnosis, 38, 60-69.

WALLACE, B. (1990b). Imagery vividness, hypnotic susceptibility, and the perception of fragmented stimuli. Journal of Personality \& Social Psychology, 58, 354-359.

(Manuscript received September 30, 1991; revision accepted for publication April 3, 1992.) 